\title{
Survival among patients with relapsed/ refractory diffuse large B cell lymphoma treated with single-agent selinexor in the SADAL study
}

\author{
Marie Maerevoet ${ }^{1 *}$ (D) Josee M. Zijlstra ${ }^{2}$, George Follows ${ }^{3}$, Rene-Olivier Casasnovas ${ }^{4}$, J. S. P. Vermaat ${ }^{5}$, \\ Nagesh Kalakonda ${ }^{6}$, Andre Goy ${ }^{7}$, Sylvain Choquet ${ }^{8}$, Eric Van Den Neste ${ }^{9}$, Brian Hill ${ }^{10}$, \\ Catherine Thieblemont ${ }^{11,12}$, Federica Cavallo ${ }^{13}$, Fatima De la Cruz ${ }^{14}$, John Kuruvilla ${ }^{15}$, Nada Hamad ${ }^{16}$, \\ Ulrich Jaeger ${ }^{17}$, Paolo Caimi ${ }^{18}$, Ronit Gurion ${ }^{19,20}$, Krzysztof Warzocha ${ }^{21}$, Sameer Bakhshi ${ }^{22}$, \\ Juan-Manuel Sancho ${ }^{23}$, Michael Schuster ${ }^{24}$, Miklos Egyed ${ }^{25}$, Fritz Offner ${ }^{26}$, Theodoros P. Vassilakopoulos ${ }^{27}$, \\ Priyanka Samal ${ }^{28}$, Matthew Ku ${ }^{29}$, Xiwen Ma ${ }^{30}$, Kelly Corona ${ }^{30}$, Kamal Chamoun ${ }^{30}$, Jatin Shah ${ }^{30}$, \\ Sharon Shacham ${ }^{30}$, Michael G. Kauffman ${ }^{30}$ and Miguel Canales ${ }^{31}$
}

\begin{abstract}
Patients with RR DLBCL who have received $\geq 2$ lines of therapy have limited treatment options and an expected overall survival (OS) of $<6$ months. The SADAL study evaluated single-agent oral selinexor in patients with RR DLBCL and demonstrated an overall response rate (ORR) of $29.1 \%$ with median duration of response (DOR) of 9.3 months. The analyses described here evaluated a number of subpopulations in order to understand how response correlates with survival outcomes in order to identify patients who could most optimally benefit from selinexor treatment. Median age was 67 years; $44.8 \%$ of patients were $\geq 70$ years of age. The median OS was 9.0 months $(95 \% \mathrm{Cl} 6.2,13.7)$ at a median follow-up of 14.8 months. The median OS was not reached in patients with a CR or PR, while patients who did not respond have a median OS of 4.9 months $(p<0.0001)$. Patients $<70$ years had an OS of 11.1 months compared with 7.8 months in patients $\geq 70$ years. Among patients with or without prior ASCT, the median OS was 10.9 and 7.8 months, respectively. Among patients with disease refractory to the most recent $D L B C L$ treatment regimen, the median OS was 7.0 months compared with 11.1 months for disease not refractory to the most recent treatment. In a patient population in which survival is expected to be $<6$ months, treatment with single-agent oral selinexor was associated with a median survival of 9 months. Increased median OS observed in patients responding to selinexor was consistent across subgroups regardless of age, prior ASCT therapy, or refractory status. Randomized studies of selinexor in combination with a variety of other anti-DLBCL agents are planned. This trial was registered at ClinicalTrials.gov (NCT02227251) on August 28, 2014. https://clinicaltrials.gov/ct2/show/NCT02227251.
\end{abstract}

Keywords: Selinexor, Exportin-1, SINE compounds, DLBCL

*Correspondence: marie.maerevoet@bordet.be

1 Service Hématologie, Institut Jules Bordet, 1000 Brussels, Belgium

Full list of author information is available at the end of the article

\section{To the editor}

Despite recent advances, nearly $50 \%$ of patients diagnosed with diffuse large B cell lymphoma (DLBCL) will succumb to their disease, with older age and comorbidities increasing risk of death and a median OS ( 6 months) with relapsing disease after $\geq 2$ prior original author(s) and the source, provide a link to the Creative Commons licence, and indicate if changes were made. The images or other third party material in this article are included in the article's Creative Commons licence, unless indicated otherwise in a credit line to the material. If material is not included in the article's Creative Commons licence and your intended use is not permitted by statutory regulation or exceeds the permitted use, you will need to obtain permission directly from the copyright holder. To view a copy of this licence, visit http://creativecommons.org/licenses/by/4.0/. The Creative Commons Public Domain Dedication waiver (http://creativeco mmons.org/publicdomain/zero/1.0/) applies to the data made available in this article, unless otherwise stated in a credit line to the data. 
therapies [1, 2]. XPO1 inhibition by selinexor, a first-inclass selective inhibitor of nuclear export compound results in cell cycle arrest; cells with DNA damage, including cancer cells, undergo apoptosis while sparing normal cells [3, 4]. Single-agent oral selinexor is approved for treatment of patients with DLBCL after $\geq 2$ prior therapies [5]. Here, we have analyzed subgroups from the SADAL trial to understand how response correlates with survival outcomes following selinexor treatment.

Overall median OS was 9.0 months after a median follow-up of 14.8 months (95\% CI: 13.2,21.7). Median OS in patients $<70$ trended longer than patients $>70$ (11.1 vs 7.8 months); HR $0.72(0.46,1.13), p=0.155$. Patients with a best response of CR or PR on selinexor had a markedly longer median OS of 29.7 months, compared to those who did not respond (4.9 months) $(p<0.0001)$ (Table 1$)$. Patients with lower baseline R-IPI (0-2) compared to R-IPI (3-5) had a significantly longer median OS (15.1 vs 4.6 months; HR $0.38[0.24,0.60], p<0.0001$ ) (Additional file 1: Figure S1). The majority (72\%) of responding patients had lower R-IPI scores at baseline.

Patients who received selinexor after ASCT compared to those who did not had a median OS of 10.9 and
7.8 months, respectively; HR 1.39 (0.85,2.28), $p=0.185$. Regarding response to most recent systemic therapy, patients who had a CR or PR trended toward longer OS (HR 0.71 [0.44, 1.17], $p=0.18$; medians 11.1 and 7 months) than those who did not respond (Fig. 1; Additional file 1: Table S2). A significantly shorter median OS was observed in patients with relapse $<1$ year from diagnosis (5.2 vs 13.1 months). Median OS was 6.6 and 10.9 months in patients that had relapsed/refractory disease $<6$ or $\geq 6$ months from last use of rituximab, respectively ( $\mathrm{HR}=1.30, p=0.30)$ (Additional file 1: Figure S2). Four patients who had not achieved CR on their most recent prior therapy achieved CR on selinexor and two patients who did not have a CR or PR to any prior therapy had a CR $(n=2)$ or PR $(n=2)$ with selinexor. Median PFS for all patients was 2.6 months (summarized for subgroups-Additional file 1: Table S1).

The median OS associated with selinexor is consistent across the majority of the analyzed subgroups and also with the novel mechanism of action and the lack of apparent cross-resistance with this and other mechanisms. Results corroborate a retrospective study on patients with RR DLBCL after ASCT, which showed that

Table 1 Overall survival in subgroups

\begin{tabular}{|c|c|c|c|c|}
\hline \multirow[t]{2}{*}{ Patients ( $n)$} & \multirow[t]{2}{*}{$\begin{array}{l}\text { All patients } \\
N=134\end{array}$} & \multirow{2}{*}{$\begin{array}{l}\text { Patients with CR or PR } \\
N=39 \\
\text { Median }(95 \% \mathrm{Cl})\end{array}$} & \multirow[t]{2}{*}{$\begin{array}{l}\text { Non-responders } \\
N=95\end{array}$} & \multirow[t]{2}{*}{ HR $(95 \% \mathrm{Cl}) ; p$ value* } \\
\hline & & & & \\
\hline All patients & & $\operatorname{NR}(29.7, N R)$ & $4.9(4.1,7.0)$ & $<0.0001$ \\
\hline \multicolumn{5}{|l|}{ Age } \\
\hline$<70(n=74)$ & $11.1(5.4,28.0)$ & $N R(N R, N R)$ & $4.9(3.1,7.0)$ & $\begin{array}{l}0.0771(0.0235,0.2527) \\
<0.0001\end{array}$ \\
\hline$\geq 70(n=60)$ & $7.8(6.1,13.7)$ & $29.7(9.1, N R)$ & $4.6(4.1,12.2)$ & $\begin{array}{l}0.1912(0.073,0.5003) \\
0.0002\end{array}$ \\
\hline \multicolumn{5}{|l|}{ Region } \\
\hline North America $(n=20)$ & $7.6(4.8,32.3)$ & $29.7(9.0,29.7)$ & $4.8(1.6,32.3)$ & $\begin{array}{l}0.2775(0.0744,1.0356) \\
0.0430\end{array}$ \\
\hline Western Europe and Australia $(n=91)$ & $10.9(6.6,15.5)$ & $N R(N R, N R)$ & $4.6(3.0,7.8)$ & $\begin{array}{l}0.0994(0.0392,0.2524) \\
<0.0001\end{array}$ \\
\hline Central and Eastern Europe and India $(n=23)$ & $6.2(5.2, N R)$ & $N R(6.2, N R)$ & $5.4(3.1, N R)$ & $\begin{array}{l}0.2248(0.028,1.8032) \\
0.1241\end{array}$ \\
\hline \multicolumn{5}{|l|}{ Baseline prognosis } \\
\hline Very good $(\mathrm{R}-\mathrm{IPI}=0)$ or good $(\mathrm{R}-\mathrm{PI}=1,2)(n=69)$ & $15.1(10.9, \mathrm{NR})$ & $\operatorname{NR}(29.7, N R)$ & $7.0(5.2,15.1)$ & $\begin{array}{l}0.1511(0.0613,0.3724) \\
<0.0001\end{array}$ \\
\hline Poor $(\mathrm{R}-\mathrm{IPI}=3,4,5)(n=58)$ & $4.6(3.0,9.0)$ & $N R(9.0, N R)$ & $4.1(2.5,5.1)$ & $\begin{array}{l}0.2176(0.0667,0.7099) \\
0.0056\end{array}$ \\
\hline \multicolumn{5}{|l|}{ Number of prior systemic treatment regimens } \\
\hline $2(n=79)$ & $9.1(5.4,15.1)$ & $N R(N R, N R)$ & $4.6(3.0,11.1)$ & $\begin{array}{l}0.131(0.0509,0.3372) \\
<0.0001\end{array}$ \\
\hline$>2(n=55)$ & $8.2(5.1,29.7)$ & $29.7(29.7, N R)$ & $4.9(3.9,7.6)$ & $\begin{array}{l}0.1389(0.0483,0.3994) \\
<0.0001\end{array}$ \\
\hline
\end{tabular}

$C l$ confidence interval, $C R$ complete response, $N R$ not reached, $O S$ overall survival, $P D$ progressive disease, $P R$ partial response, $S D$ stable disease

${ }^{*} \mathrm{HR}$ and $p$ value is comparing responder versus non-responder 

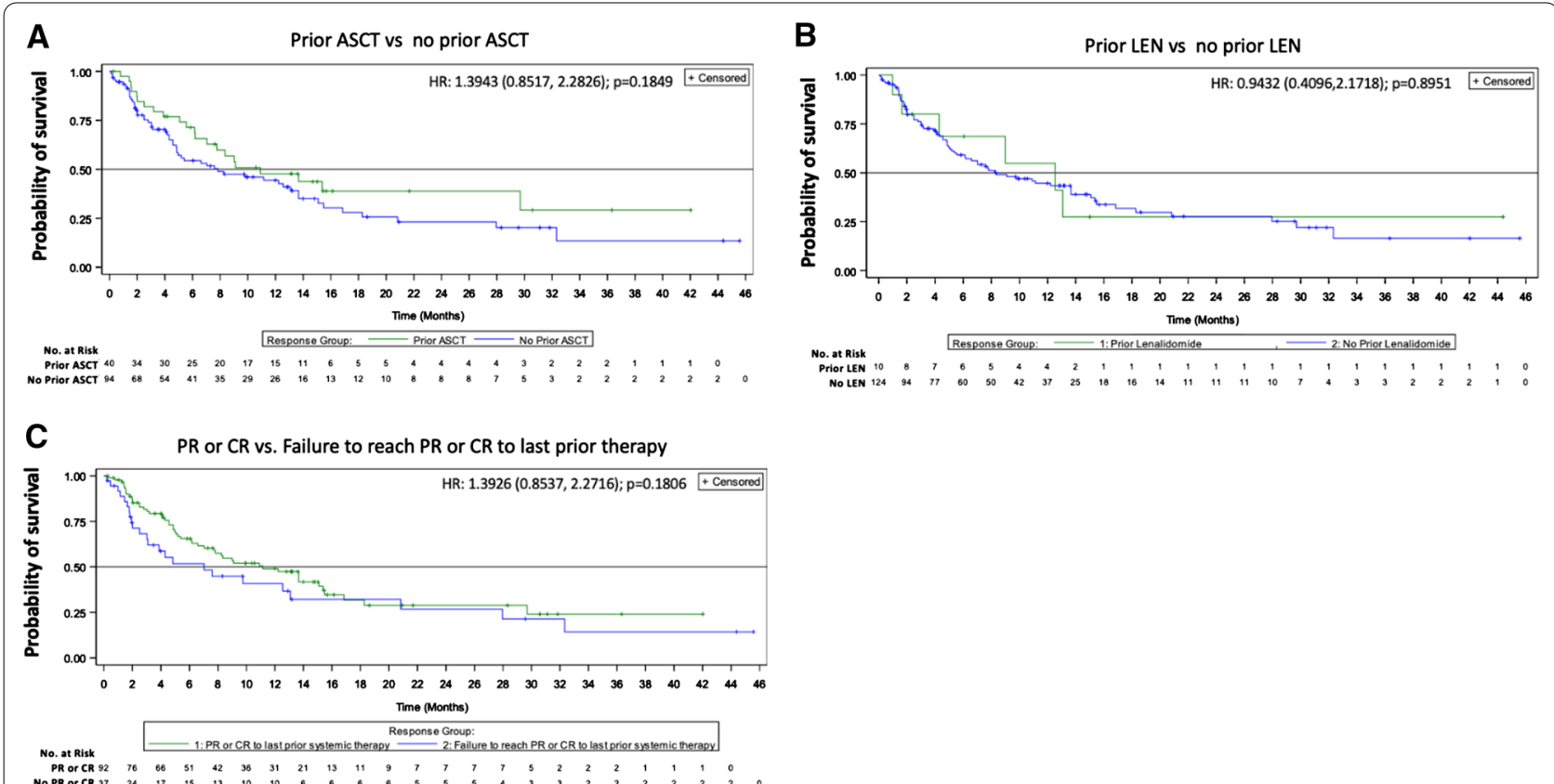

Fig. 1 Overall survival by prior regimens and response. Kaplan-Meier curves according to A prior ASCT status; B prior use of lenalidomide; and C response to prior therapy: $P R$ or $C R$ and failure to reach $P R$ or $C R$

the median OS was 6.6 months with cytotoxic chemotherapy compared to 11.3 months with novel agents [6]. While combination tafasitamab and lenalidomide showed an $\sim 60 \%$ ORR for relapsed/refractory DLBCL and median OS was NR after a median follow-up of 19.6 months [7], it is difficult to compare to SADAL since $50 \%$ of patients had only one prior line of therapy compared to $3 \%$ on SADAL. In addition, only $18 \%$ of patients had primary refractory disease (relapse $<6$ months of frontline therapy) compared to $47 \%$ of patients with available data on SADAL. Furthermore, $44 \%$ were refractory (i.e., relapsed $<6$ months) to most recent therapy compared to $66.4 \%$ of SADAL patients. Further demonstration of the efficacy and safety consistent with the novel mechanism of action and lack of cross-resistance is observed with combination treatment of selinexor with backbone chemotherapy: 100\% ORR with $90 \%$ CR using $\mathrm{R}-\mathrm{CHOP}+$ selinexor as frontline treatment for DLBCL and follicular lymphoma [8] and 78\% ORR using $\mathrm{R}-\mathrm{ICE}+$ selinexor for relapsed/refractory DLBCL [9]. These combination results are consistent with a significant anti-DLBCL contribution of selinexor to standard chemotherapy and are being further evaluated.

Single-agent oral selinexor treatment was associated with a longer OS than expected based on contemporary case series [10-12] despite patient treatment, response history, age, and comorbidities. Given the beneficial impact of selinexor as a single agent and the poor prognosis of many patients, randomized studies of selinexor in combination with a variety of other anti-DLBCL agents are planned. Taken together, selinexor represents a safe, orally available option for patients whose disease has relapsed or is refractory to $\geq 2$ prior therapies, including patients $>70$ years old or those with significant comorbidities.

\section{Abbreviations}

ASCT: Autologous stem cell transplant; CR: Complete response; DLBCL: Diffuse large B cell lymphoma; HR: Hazard ratio; ORR: Overall response rate; OS: Overall survival; PR: Partial response; R-CHOP: Rituximab and cyclophosphamide, doxorubicin, vincristine, and prednisone; R-ICE: Rituximab and ifosfamide, carboplatin, etoposide; R-IPI: Revised International Prognostic Index; RR: Relapsed/refractory; XPO1: Exportin-1.

\section{Supplementary Information}

The online version contains supplementary material available at https://doi. org/10.1186/s13045-021-01122-1.

Additional file 1. Supplemental Material.

\section{Acknowledgements}

JetPub Scientific Communications, LLC supported by funding from Karyopharm, provided drafts and editorial assistance to the authors during preparation of this manuscript.

\section{Authors' contributions}

MM, JMZ, GF, ROC, JSPV, NK, AG, SC, EVDN, BH, CT, FC, FDLC, JK, NH, UL, PC, $R G, K W, S B, J M S, M S, M E, F O, T V, P S, M K, J S, S S, M G K$, and MC collected the data. MM, JS, SS, and MGK contributed to the study design. XM analyzed the data. All authors interpreted the data. All authors read and approved the final manuscript. 


\section{Funding}

This study was supported by Karyopharm Therapeutics.

\section{Availability of data and materials}

Karyopharm Therapeutics agrees to share individual participant data that underlie the results reported in this article (after deidentification), including the study protocol and statistical analysis plan. Data availability will begin 9 months after publication and will be available 36 months after publication. To gain access, data requestors should submit a proposal to medicalinformation@karyopharm.com. Proposals will be reviewed by an independent review committee identified for this purpose.

\section{Declarations}

\section{Ethics approval and consent to participate}

The study was approved and performed in accordance with the International Conference on Harmonization, the Guidelines for Good Clinical Practice, appropriate regulatory requirements, and with approval of institutional review boards at individual enrolling institutions. All patients provided written informed consent before study start.

\section{Consent for publication}

Not applicable.

\section{Competing interests}

GF reports personal fees from Karyopharm and Roche, outside the submitted work. R-OC reports grants, personal fees, and non-financial support from Roche, personal fees and non-financial support from Takeda, BMS, Amgen, Janssen, Abbvie, grants and personal fees from Gilead, and personal fees from Merck, outside the submitted work. NK reports research support from Verastem, Gilead, Celgene, and Roche, as well as honoraria from Gilead, Janssen, and Karyopharm. AG reports personal fees and honoraria from AstraZeneca, personal fees and board membership from Cota and Kite/Gilead, personal fees from Janssen, Celgene, Acerta, and research funding from Constellation, Bayer, CALBG, Genentech, Hoffman-La Roche, MD Anderson, Morphosys, Pharmacyclics, and the University of Nebraska, outside the submitted work. BH reports grants and personal fees from Karyopharm, outside the submitted work. FC reports personal fees from Takeda, Gilead, and Janssen, outside the submitted work. JK reports research support from Canadian Cancer Society, Leukemia and Lymphoma Society Canada, Princess Margaret Cancer Foundation, Janssen, Roche, Astra Zeneca, consultation fees from Abbvie, BMS, Gilead, Karyopharm, Merck, Roche, Seattle Genetics, honoraria from Amgen, Antengene, Astra Zeneca, BMS, Gilead, Incyte, Janssen, Karyopharm, Merck, Novartis, Pfizer, Roche, Seattle Genetics, TG Therapeutics, scientific advisory board member for Lymphoma Canada (Chair) and on the data safety monitoring board for Karyopharm Therapeutics. UJ reports personal fees from Karyopharm, during the conduct of the study; grants and personal fees from AbbVie, Celgene, Gilead, Janssen, Novartis, Roche, Takeda, Amgen, Miltenyi, and BMS, outside the submitted work. JMS reports honoraria from Roche, Janssen, Gilead, Celgene, and Novartis, outside the submitted work. MS reports personal fees from Karyopharm during the conduct of the study, and personal fees from Amgen, Abbvie, Gilead, Takeda, Celgene, Pharmacyclics, Astellas, Verastem, Merck, Novartis, Genentech, and Seattle Genetics, outside the submitted work. TPV reports honoraria from WinMedica, Astellas, and Gilead, honoraria, advisory board membership and research support from Takeda, honoraria and advisory board membership from Roche, Bristol, Genesis, and Novartis, advisory board membership at Janssen, honoraria and research support from Merck and Amgen, and research support from Pfizer and Karyopharm. XM, KC, JS, SS, and MK are employees of Karyopharm. MK and SS are stockholders of Karyopharm. SS holds patents (8999996, 9079865, 9714226, PCT/US12/048319, and 1574957) on hydrazide containing nuclear transport modulators and uses, and pending patents (PCT/US12/048319, 499/2012, PI20102724, and 2012000928) on hydrazide-containing nuclear transport modulators and uses. MC reports personal fees from Celgene, Gilead, Janssen, Karyopharm, Novartis, Roche, Sandoz, and Servier, outside the submitted work. All other authors declare no competing interests.

\section{Author details}

'Service Hématologie, Institut Jules Bordet, 1000 Brussels, Belgium.

${ }^{2}$ Amsterdam UMC, Vrije Universiteit, Cancer Center, Amsterdam, Netherlands.
${ }^{3}$ Addenbrooke's Hospital, Cambridge, UK. ${ }^{4}$ Hématologie Clinique and INSERM 1231, CHU Dijon, Dijon, France. ${ }^{5}$ LUMC, Leiden, Netherlands. ${ }^{6}$ University of Liverpool, Liverpool, UK. ${ }^{7}$ Hackensack University Medical Center, Hackensack, USA. ${ }^{8}$ Hôpital Pitié Salpêtrière, Paris, France. ${ }^{9}$ Cliniques Universitaires Saint-Luc, Brussels, Belgium. ${ }^{10} \mathrm{Cleveland}$ Clinic, Cleveland, USA. ${ }^{11} \mathrm{APHP}$, Hemato-oncology, Saint-Louis Hospital, Paris, France. ${ }^{12}$ Diderot University, Paris, France. ${ }^{13}$ University of Torino, Turin, Italy. ${ }^{14} \mathrm{Hospital}$ Universitario Virgen del Rocio, Sevilla, Spain. ${ }^{15}$ Princess Margaret Cancer Centre, Toronto, Canada. ${ }^{16} \mathrm{St}$. Vincent's Hospital Sydney, Darlinghurst, Australia. ${ }^{17}$ Medical University of Vienna, Vienna, Austria. ${ }^{18}$ UH Seidman Cancer Center, Cleveland, USA. ${ }^{19}$ Rabin MC, Petah Tiqwa, Israel. ${ }^{20}$ Tel Aviv University, Tel Aviv, Israel. ${ }^{21}$ Instytut Hematologii I Transfuzjologii, Warsaw, Poland. ${ }^{22}$ Dr. B. R. A. Institute Rotary Cancer Hospital, New Delhi, India. ${ }^{23}$ Hospital Universitari Germans Trias I Pujol, Barcelona, Spain. ${ }^{24}$ Stony Brook University Hospital Cancer Center, Stony Brook, USA. ${ }^{25}$ Teaching Hospital Mór Kaposi, Kaposvár, Hungary. ${ }^{26}$ UZ Gent, Gent, Belgium. ${ }^{27}$ Laikon General Hospital, National and Kapodistrian University of Athens, Athens, Greece. ${ }^{28}$ Institute of Medical Sciences and SUM Hospital, Bhubaneswar, Odisha, India. ${ }^{29}$ St.Vincent's Hospital Melbourne, Fitzroy, Australia. ${ }^{30}$ Karyopharm Therapeutics, Newton, USA. ${ }^{31}$ Hospital Universitario La Paz, Madrid, Spain.

Received: 20 April 2021 Accepted: 1 July 2021

Published online: 16 July 2021

\section{References}

1. Hedström G, Hagberg O, Jerkeman M, Enblad G. The impact of age on survival of diffuse large B-cell lymphoma-a population-based study. Acta Oncol (Madr). 2015;54:916-23.

2. Morrison VA, Hamlin P, Soubeyran P, Stauder R, Wadhwa P, Aapro M, et al. Approach to therapy of diffuse large B-cell lymphoma in the elderly: the International society of geriatric oncology (SIOG) expert position commentary. Ann Oncol. 2015;26:1058-68.

3. Senapedis WT, Baloglu E, Landesman Y. Clinical translation of nuclear export inhibitors in cancer. Semin Cancer Biol. 2014;27:74-86.

4. Lapalombella R, Sun Q, Williams K, Tangeman L, Jha S, Zhong Y, et al. Selective inhibitors of nuclear export show that CRM1/XPO1 is a target in chronic lymphocytic leukemia. Blood. 2012;120:4621-34.

5. Kalakonda N, Maerevoet M, Cavallo F, Follows G, Goy A, Vermaat JSP, et al. Selinexor in patients with relapsed or refractory diffuse large B-cell lymphoma (SADAL): a single-arm, multinational, multicentre, open-label, phase 2 trial. Lancet Haematol. 2020;7:e511-22.

6. Nagle SJ, Woo K, Schuster SJ, Nasta SD, Stadtmauer E, Mick R, et al. Outcomes of patients with relapsed/refractory diffuse large B-cell lymphoma with progression of lymphoma after autologous stem cell transplantation in the rituximab era. Am J Hematol. 2013;88:890-4.

7. Salles G, Duell J, González Barca E, Tournilhac O, JurczakW, Liberati AM, et al. Tafasitamab plus lenalidomide in relapsed or refractory diffuse large B-cell lymphoma (L-MIND): a multicentre, prospective, single-arm, phase 2 study. Lancet Oncol. 2020;21:978-88.

8. Seymour E. Selinexor in combination with R-CHOP for frontline treatment of non-Hodgkin lymphoma: results of a phase 1b study. Blood. 2020;136:11-2.

9. Rutherford S. A phase I study of selinexor and R-ICE in patients with relapsed/refractory aggressive B-cell lymphomas. ASH; 2020.

10. Dimou M, Papageorgiou SG, Stavroyianni N, Katodritou E, Tsirogianni M, Kalpadakis C, et al. Real-life experience with the combination of polatuzumab vedotin, rituximab and bendamustine in aggressive B-cell lymphomas. Hematol Oncol. 2021.

11. Wang S, Wang L, Hu J, Qian W, Zhang X, Hu Y, et al. Outcomes in refractory diffuse large B-cell lymphoma: results from a multicenter real-world study in China. Cancer Commun. 2021:41:229-39.

12. Di Rocco A, Cuneo A, Di Rocco A, Merli F, De Luca G, Petrucci L, et al. Relapsed/refractory diffuse large B-cell lymphoma patients. A multicenter retrospective analysis of eligibility criteria for car-T cell therapy. Leuk Lymphoma. 2020;62:828-36.

\section{Publisher's Note}

Springer Nature remains neutral with regard to jurisdictional claims in published maps and institutional affiliations. 